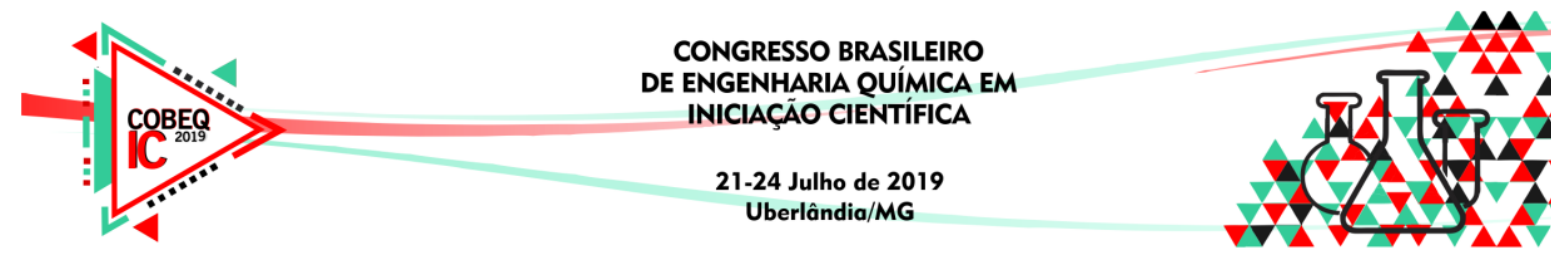

\title{
PURIFICAÇÃO DE COMPOSTOS BIOATIVOS PRESENTES NO PEQUI (Caryocar brasiliense Camb.) POR ULTRAFILTRAÇÃO
}

\author{
M. S. M. SA ${ }^{1}$, F. S. MAGALHÃES ${ }^{1}$, V. L. CARDOSO ${ }^{1}$ e M. R. M. REIS ${ }^{1}$ \\ ${ }^{1}$ Universidade Federal de Uberlândia, Faculdade de Engenharia Química \\ E-mail para contato: miria@ufu.br
}

\begin{abstract}
RESUMO - A busca por maneiras de enriquecer alimentos através de fontes naturais tem crescido nos últimos anos. O pequi é uma fruta do cerrado brasileiro, que apresenta compostos fenólicos substanciais que justificam o processamento dessa fruta. O objetivo deste trabalho é a clarificação e a concentração de compostos bioativos do extrato aquoso de pequi utilizando ultrafiltração. $\mathrm{O}$ extrato aquoso de pequi foi preparado a uma concentração de $25 \mathrm{~g}$ de polpa em $100 \mathrm{~mL}$ de água destilada, filtrado em papel de filtro, centrifugado a $8000 \mathrm{rpm}$ por 10 minutos e em seguida ultrafiltrado a 6 bar de pressão através de uma membrana de polietersulfona plana de $5 \mathrm{kDa}$ com área efetiva de filtração de 0,00193 $\mathrm{m}^{2}$ (Microdyn-Nadir). O processo de ultrafiltração reduziu o teor de lipídeos $(99,07 \%)$, sólidos totais $(79,13 \%)$, sólidos solúveis $(55,83 \%)$ e concentrou o teor de compostos fenólicos totais $(72,86 \%)$ que justifica a eficácia do processo na retenção de moléculas maiores, como os compostos fenólicos. Foi possível perceber que a membrana de $5 \mathrm{kDa}$ foi eficiente para concentrar compostos bioativos e também para retenção de partículas maiores.
\end{abstract}

\section{INTRODUÇÃO}

O Cerrado brasileiro, segundo maior bioma do país (IBGE, 2004), possui diversos frutos nativos com potencial de exploração científica e econômica, como o Pequi (Caryocar brasiliense Camb.). Esse fruto é rico em proteínas, vitaminas compostos bioativos e antioxidantes, como os compostos fenólicos, podendo ser utilizados na produção de fármacos e enriquecimento de alimentos (Sarkis, 2014; Roesler et al., 2007; Sa et al., 2017). No entanto, o processamento desta fruta ainda é limitado, que pode ser atribuído à dificuldade no manuseio, por possuir espinhos no seu interior (Lima, 2008). A valorização dos frutos nativos de cada região, aliando seu uso na fruticultura, é uma alternativa de geração de renda para as populações locais (Oliveira et al., 2017) e uma maneira de preservação ambiental em projetos de restauração de áreas degradadas (Beuchle et al., 2015).

De maneira geral, a concentração de extratos oriundos de frutos é frequentemente realizada em processos com aplicação de calor, que podem danificar compostos termolábeis presentes no fruto do pequi. Uma alternativa a esses processos convencionais são os processos físicos, como centrifugação e processos de separação por membranas que são menos agressivos e eficientes (Li e Chase, 2010). Machado et al. (2015) concentraram o extrato aquoso de pequi pelo processo de ultrafiltração (UF) e obtiveram $65 \%$ e $98 \%$ de compostos fenólicos e 


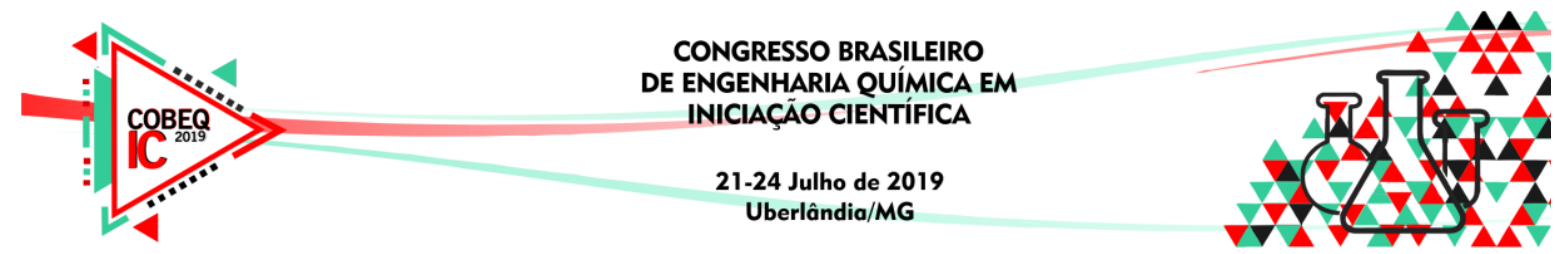

carotenoides, respectivamente, no concentrado. Cissé et al. (2011) retiveram $80 \%$ de antocianina aplicando uma membrana de UF, mostrando a eficiência que esse processo tem em concentrar não só compostos bioativos mas também, açúcares (Alles et al., 2015). Assim, este trabalho propõe a ultrafiltração do tipo dead-end para a concentração de compostos bioativos presentes no extrato de pequi.

\section{MATERIAIS E MÉTODOS}

\subsection{Processo de Extração}

O extrato aquoso de pequi foi preparado a partir da adição de $25 \mathrm{~g}$ de polpa em $100 \mathrm{~mL}$ de água destilada, sob agitação magnética durante $1 \mathrm{~h}$ a $80{ }^{\circ} \mathrm{C}$ (Magalhães et al., 2018). Após esse processo, o extrato aquoso foi centrifugado a $8000 \mathrm{rpm}$ por $10 \mathrm{~min}$ para a retirarada de óleo sólidos.

\subsection{Processo de Separação por Membrana}

Para o processo de UF, utilizou-se um módulo de filtração de capacidade de $70 \mathrm{~mL}$, onde foi adicionado $60 \mathrm{~mL}$ de solução na célula e esta foi pressurizada a 6 bar utilizando-se nitrogênio. Foi utilizada uma membrana de polietersulfona plana de $5 \mathrm{kDa}$ com área efetiva de filtração de $0,00193 \mathrm{~m}^{2}$ (Microdyn-Nadir). O extrato centrifugado foi ultrafiltrado até atingir um fator de concentração igual a $2(\mathrm{Fc}=2,0)$.

\subsection{Análises Físico-Químicas}

As amostras de extrato centrifugado (EC) e de seu permeado após a ultrafiltração (ECU) foram caracterizadas de acordo com o teor de fenólicos totais (TPC) determinado pelo método de Folin-Ciocalteau, descrito por Swain e Hillis (1959), teor de sólidos totais (TSC) foi encontrado colocando-se $2 \mathrm{~mL}$ de amostra em placas de petri e pesadas antes e após secagem em estufa a $105^{\circ} \mathrm{C}$ por $24 \mathrm{~h}$, sólidos solúveis (SS) estipulados através de um refratômetro digital e o teor de lipídeos determinado pela metodologia sugerida por Bligh and Dyer (1959). Todas as análises foram feitas em triplicata e analisadas estatisticamente através de um Teste de Tukey com significância de 5\%.

As diferenças entre médias foram avaliadas pela análise de variância (ANOVA), utilizando o teste de Tukey, com significância de 5\% com auxílio do software Sisvar® 5.6.

\section{RESULTADOS E DISCUSSÃO}

O processo de ultrafiltração teve duração de 165 minutos e o fluxo estabilizado foi de cerca de $5 \mathrm{~L} \mathrm{~h}^{-1} \mathrm{~m}^{-2}$, o que pode ser verificado na Figura 1 . Nota-se que nos primeiros 20 minutos há um decaimento mais acentuado do fluxo que pode ser atribuído à deposição de sólidos na camada superficial da membrana, característica de filtrações do tipo dead-end (Bacchin et al., 2005). 


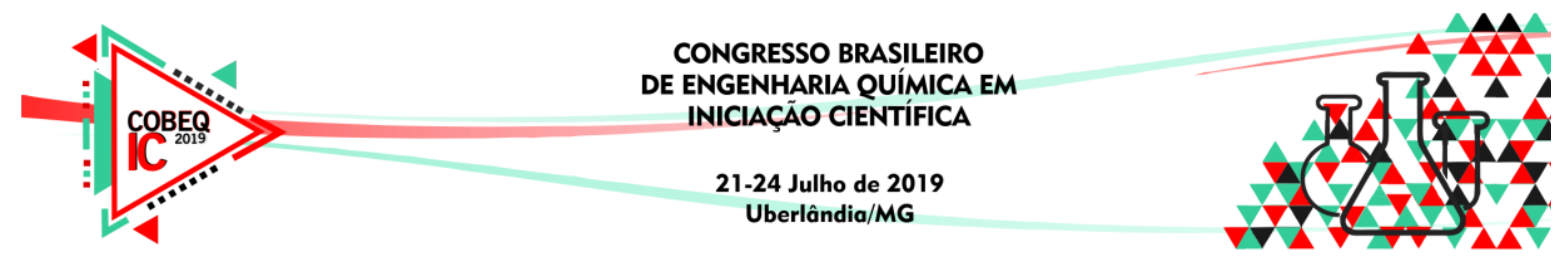

Figura 1 - Fluxo de permeado por tempo de filtração do extrato de pequi

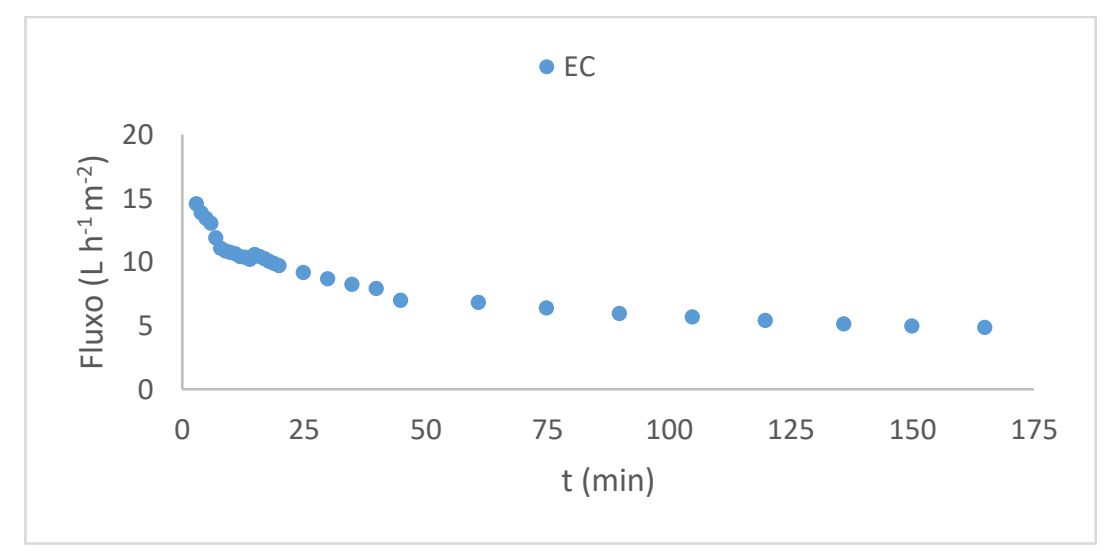

As características físico-químicas da polpa de pequi e do extrato aquoso antes e após o processo de ultrafiltração proposto podem ser vistos na Tabela 2.

Tabela 1 - Características físico-químicas do extrato aquoso de pequi centrifugado (EC) e ultrafiltrado (ECU)

\begin{tabular}{|c|c|c|c|c|}
\hline Amostra & $\begin{array}{c}\text { TPC }(\mathrm{mgAG} \\
\left.100 \mathrm{~g} \mathrm{polpa}^{-1}\right)\end{array}$ & $\begin{array}{c}\text { TSC } \\
\left(\mathrm{g} \mathrm{L}^{-1}\right)\end{array}$ & $\begin{array}{c}\text { SS } \\
\left({ }^{\circ} \mathrm{Brix}\right)\end{array}$ & $\begin{array}{c}\text { Teor de Lipídeos } \\
(\%)\end{array}$ \\
\hline EC & $108,56^{\mathrm{a}} \pm 0,44$ & $20,6^{\mathrm{a}} \pm 0,39$ & $1,20^{\mathrm{a}} \pm 0,00$ & $1,07^{\mathrm{a}} \pm 0,20$ \\
\hline ECU & $29,46^{\mathrm{b}} \pm 1,33$ & $4,30^{\mathrm{b}} \pm 0,25$ & $0,53^{\mathrm{b}} \pm 0,06$ & $0,01^{\mathrm{b}} \pm 0,003$ \\
\hline
\end{tabular}

*Letras diferentes em uma mesma coluna indicam diferença estatística significativa entre as amostras $(\mathrm{p} \leq 0,05)$.

O extrato permeado apresentou redução no teor de sólidos totais, superior a $79 \%$ e uma diminuição dos valores de compostos fenólicos $(72,86 \%)$, sólidos solúveis $(55,83 \%)$ e teor de lipídeos $(99,07 \%)$. Baklouti et al. (2012) na ultrafiltração do suco de romã, utilizando uma membrana de $5 \mathrm{kDa}$, notaram uma redução de $55 \%$ no teor de compostos fenólicos no permeado, o que mostra que a ultrafiltração em uma membrana de $5 \mathrm{kDa}$. Machado; Mello e Hubinger, (2015) estudaram a concentração de compostos fenólicos da polpa de pequi e conseguiram reter $65 \%$ dos compostos fenólicos utilizando uma membrana de ultrafiltração de $100 \mathrm{kDa}$. A ultrafiltração foi capaz de remover quase $100 \%$ da quantidade de lipídeos presente no extrato, evidenciando a eficácia na clarificação do extrato de pequi, resultados semelhantes foram obtidos por Magalhães et al. (2019).

\section{CONCLUSÃO}

O processo de ultrafiltração através de uma membrana de $5 \mathrm{kDa}$ foi capaz de reter os compostos bioativos presentes no extrato de pequi, haja visto que foi retido no concentrado cerca de $73 \%$ de TPC, evidenciando a eficiência da ultrafiltração em concentrar compostos bioativos. Além disso, observou-se também a clarificação do extrato aquoso de pequi, alcançando uma redução no teor de lipídeos superior a 99\% e redução do teor de sólidos totais de $79,13 \%$. Tanto a retenção de compostos quanto a clarificação do extrato podem ser atribuídas 


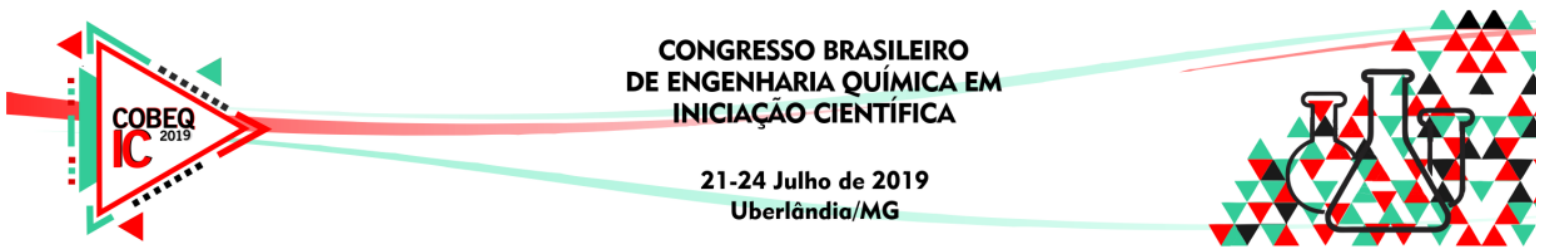

à formação de torna na membrana, que retêm os compostos fenólicos que se ligam a outros sólidos em suspensão e consequentemente a maior parte dos sólidos.

\section{REFERÊNCIAS}

ALLES, M.J.L., TESSARO, I.C., NOREÑA, C.P.Z. Concentration and purification of Yacon (Smallanthus sonchifolius) root fructooligosaccharides using membrane technology. Food Technol. Biotechnol. 53 (2), 190-200. (2015).

BACCHIN, YOLAINE BESSIERE, BRUCE JEFFERSON. Dead-end filtration of natural organic matter: experimental evidence of critical conditions. Desalination, Elsevier, 175 (1), pp.29-36. (2005).

BAKLOUTI, S., ELLOUZE-GHORBEL, R., MOKNI, A., CHAABOUNI. Clarification of pomegranate juice by ultrafiltration: Study of juice quality and of the fouling mechanism. Fruits, 67(3), 215-225. doi:10.1051/fruits/2012010. (2012).

BEUCHLE, R.; GRECCHI, R.C.; SHIMABUKURO, Y.E.; SELIGER, R.; EVA, H.D.; SANO, E.; ACHARD, F. Land cover changes in the Brazilian Cerrado and Caatinga biomes from 1990 to 2010 based on a systematic remote sensing sampling approach. Applied Geography, Amsterdam, v. 58,p.116-127, mar. (2015).

BISWAS, P. P., MONDAL, M. AND DE, S. Primary Clarification of Bottle Gourd Juice. Journal of Food Processing and Preservation, 40: 226-238. doi:10.1111/jfpp.12599. (2016).

BLIGH, E. G.; DYER, W. J. A rapid method of total lipid extration and purification. Canadian Journal of Biochemistry and Physiology, v. 37, n. 8, p. 911-917. (1959).

BRIAO, V. B.; TAVARES, C. R. G. Nota Científica: ultrafiltração de efluente da indústria de laticínios para recuperação de nutrientes: efeito da pressão e da velocidade tangencial. Braz. J. Food Technol., Campinas , v. 15, n. 4, p. 352-362, dez. (2012).

CISSÉ, M., VAILlANT, F., PALLET, D., DORNIER, M. Selecting ultrafiltration and nanofiltration membranes to concentrate anthocyanins from roselle extract (Hibiscus sabdariffa L.), Food Research International, Volume 44, Issue 9, 2011, Pages 26072614, ISSN 0963-9969. (2011).

IBGE. Disponível em: <http://mapas.ibge.gov.br/biomas2/viewer.htm>. Acesso em: 23/03/2019. (2004).

LI, J.; CHASE, H. A. Applications of membrane techniques for purification of natural products. Biotechnology Letters, 32, 601-608. (2010).

LIMA, A. Caracterização química, avaliação da atividade antioxidante in vitro e in vivo, e identificação dos compostos fenólicos presentes no Pequi (Caryocar brasiliense, Camb.). 


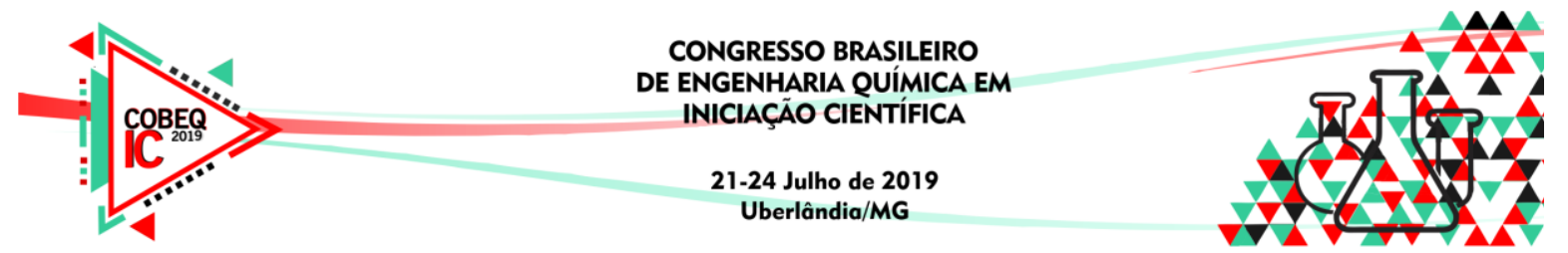

Tese (Doutorado em Bromatologia) - Faculdade de Ciências Farmacêuticas, University of São Paulo, São Paulo, 2008. (2008).

MACHADO, M.T.C., MELLO, B.C., HUBINGER, M.D. Evaluation of pequi(Caryocar Brasiliense Camb.) aqueous extract quality processed by membranes. Food and Bioproducts Processing, 95, 304-312. (2015).

MACHADO, M.T.C., TREVISAN, S., PIMENTEL-SOUZA, J.D.R., PASTORE, G.M., HUBINGER,M.D. Clarification and concentration of oligosaccharides from artichoke extractby a sequential process with microfiltration and nanofiltration membranes. Journal of Food Engineering, 180, 120-128. (2016).

MAGALHÃES, F.S. Purificação de extrato de pequi (Caryocar brasiliense Camb.) utilizando coagulantes naturais e processo de separação por membranas - Uberlândia. 2018. 109 f. Dissertação (Mestrado em Engenharia Química) - Universidade Federal de Uberlândia, Uberlândia. (2018).

MAGALHÃES, F.S., CARDOSO, V.L., REIS, M. H. M. Sequential process with bioadsorbents and microfiltration for clarification of pequi (Caryocar brasilienseCamb.) fruit extract. Food and Bioproducts Processing, 108, 105-116. (2018).

MAGALHÃES, F.S.; SÁ, M.S.M.; CARDOSO, V.L.; REIS, M.H.M. Recovery of phenolic compounds from pequi (Caryocar brasiliense Camb.) fruit extract by membrane filtrations: Comparison of direct and sequential processes. JOURNAL OF FOOD ENGINEERING, v. 257, p. 26-33, 2019.

OLIVEIRA, C.S.; GONÇALVES, L.E.N.; COUTINHO, M.P.; PEIXOTO, N.; GATTO, A. Aspectos Socioambientais da Comercialização de Pequi em Goiás. Floresta Ambient., Seropédica, v. 24, ne00058213. (2017).

ROESLER, R., MALTA, L.G., CARRASCO, L.C., HOLANDA, R.B, SOUSA, C.A.S., PASTORE, G.M. Antioxidant activity of cerrado fruits. Ciência e Tecnologia de Alimentos, 27, 53-60 (2007).

SA, M. S. M.; MAGALHÃES, F. S.; CARDOSO, V. L.; REIS, M. H. M. "Otimização das condições para extração aquosa de compostos fenólicos da polpa de pequi (Cariocar brasiliense Camb.)", p. 498-503. (2017).

SARKIS, J. R. Extração de compostos bioativos de tortas de nozes e sementes e aplicação de tecnologias elétricas no gergelim - Tese (Doutorado em Engenharia Química) Departamento de Engenharia Química - Universidade federal do Rio Grande do Sul, Porto Alegre. (2014).

SOUSA, L. S. Extração e purificação dos compostos fenólicos presentes nas folhas de Camellia sinensis. Dissertação de mestrado. Universidade Federal de Uberlândia. Faculdade de Engenharia Química. Uberlândia (2016). 


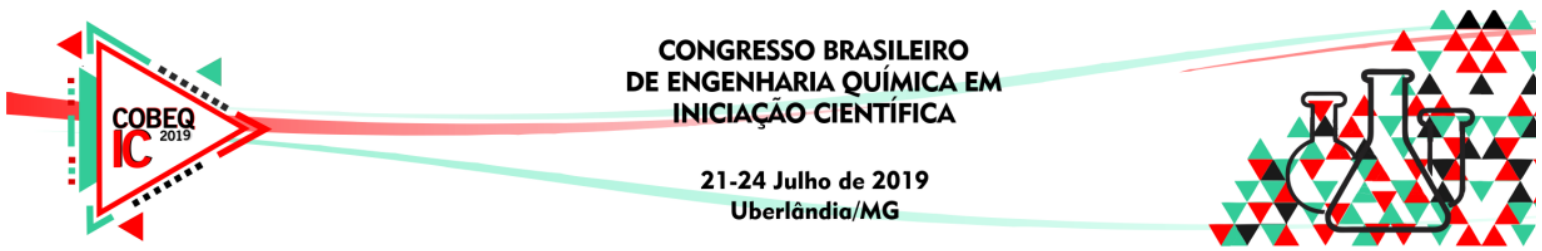

SWAIN, T.; HILLIS, W.E.; The phenolic constituents of Prunus domestica. The quantitativeanalysis of phenolic constituents. Journal of the Science of Food and Agriculture, v.10, p.63-68. (1959). 